\title{
Impact de la sécheresse \\ et de la dégradation des aires \\ protégées sur la répartition \\ des trypanosomoses bovines et de leurs vecteurs dans le bassin versant de l'Oti au nord du Togo
}

\author{
B. Dao ${ }^{1 *}$ G. Hendrickx ${ }^{2}$ I. Sidibé ${ }^{1}$ \\ A.M.G. Belem ${ }^{3}$ S. De La Rocque ${ }^{4}$
}

Mots-clés

Bovin - Glossina - Trypanosomose Sécheresse - Déboisement - Système d'information géographique - Togo.

\begin{abstract}
Résumé
Logé dans I'aire de transition entre les zones semi-aride au Nord et humide au Sud, le bassin versant de l'Oti (BVO) a subi durant les décennies 1970 1990 de très fortes pressions anthropiques et climatiques. La sécheresse, I'avancée du désert et les activités économiques reposant essentiellement sur l'agriculture et l'élevage ont contribué à la dégradation de ce bassin. En outre, de par sa position, le BVO accueille chaque année pendant la saison sèche du bétail transhumant venant du Burkina Faso et du Niger. Ce mouvement saisonnier des bovins transhumants a été source de maintien et de transmission de plusieurs maladies transfrontalières et des trypanosomoses animales. Des données de synthèse bibliographique et d'enquêtes de terrain effectuées en fin de saison sèche en avril et mai 2006, traitées dans un système d'information géographique (SIG), ont permis d'évaluer l'impact des facteurs anthropiques et climatiques sur l'épidémiologie des trypanosomoses animales dans le BVO. Les résultats de l'étude ont montré que le BVO était fortement dégradé ; les grands mammifères sauvages et les glossines ont quasiment disparu de la réserve de faune de la Fosse aux lions. Glossina tachinoides a été la seule espèce capturée au nord du $10^{\mathrm{e}}$ parallèle de latitude N. Trypanosoma vivax a été classé comme le principal parasite responsable des trypanosomoses bovines. Il a été conclu que I'homme par son action sur l'environnement et les changements climatiques ont provoqué un recul vers le sud de l'aire de répartition des glossines.
\end{abstract}

\section{INTRODUCTION}

Le concept juridique de « zones protégées » a été introduit en Afrique occidentale française en 1925. En application à ce concept, le gouverneur général du Togo, pour empêcher une grande déforestation du pays, a pris un arrêté créant de vastes domaines forestiers

1. Cirdes, Bobo-Dioulasso, Burkina Faso

2. Avia - GIS, Zoersel, B-2980 Belgium.

3. IDR/UPB, Ouagadougou, Burkina Faso.

4. FAO, Rome, Italie.

* Auteur pour la correspondance

Institut togolais de recherche agronomique, BP 1163, Lomé, Togo.

Tél. : +228252148; fax : +228251559

E-mail : balabadidao@yahoo.fr classés (3). Ces domaines sont demeurés, autant que possible, à l'écart de tout contact humain. La pénétration et la chasse ont été interdites $(4,15,27,28,33)$. Le souci de conservation du patrimoine faunistique et végétal a conduit le Gouvernement Togolais à agrandir certaines aires et en ériger d'autres en parcs nationaux (33).

Dans l'extrême nord du Togo, toute la vallée de l'Oti a été mise sous protection en 1981, créant la réserve de faune Oti - Mandouri d'une superficie de $1878,40 \mathrm{~km}^{2}(1,4)$. Ces aires, souvent maintenues manu militari, ont, certes, permis de préserver les formations faunistiques et végétales, mais ont privé la population agricole riveraine des terres arables. Elles ont en outre provoqué une surexploitation et un surpâturage des zones non interdites entraînant ainsi un appauvrissement et une dégradation des sols et du couvert végétal. Durant les années 1990, suite à la crise sociopolitique consécutive à l'avènement de la démocratie, le système de protection militaire 
s'est affaibli. C'est ainsi que les populations ont envahi partiellement ou totalement les aires protégées en exploitant d'une manière anarchique et illicite les ressources forestières et fauniques $(2,33)$.

Bien qu'offrant des atouts à l'agriculture et à l'élevage, les terres ré-envahies hébergent des mouches tsé-tsé, vecteurs des trypanosomoses humaines et animales. Ces insectes sont étroitement liés à la végétation (9). La dégradation de cette dernière souvent due à l'action anthropique et climatique entraîne des répercutions négatives aussi bien sur la densité des glossines que sur la distribution des hôtes $(6,13,18,19,32)$.

La présente étude, entreprise seize ans après la ré-invasion partielle ou totale des aires protégées, a eu pour objectif de déterminer l'impact des facteurs anthropiques et climatiques sur la répartition des trypanosomoses bovines, de leurs vecteurs, et de leurs hôtes.

\section{MATERIEL ET METHODES}

La zone d'étude (figure 1 ) est localisée entre $0^{\circ}$ et $1^{\circ}$ de long. E, et $10^{\circ}$ et $11^{\circ}$ de lat. N. D'une superficie de $8533 \mathrm{~km}^{2}$, un tiers de la zone d'étude est occupé par des réserves de flore et de faune. Le fleuve Oti et ses affluents constituent le principal réseau hydrographique. Le climat est subhumide, de type tropical continental avec une seule saison sèche très marquée d'octobre à avril, suivie d'une saison de pluie le reste de l'année. Durant la saison sèche, l'hygrométrie descend en dessous de 15 p. 100 et l'harmattan souffle de novembre à février. La pluviométrie moyenne annuelle est de 1000 $\mathrm{mm}$ mais elle présente des variations interannuelles importantes. Elle est mal repartie dans le temps et dans l'espace, et le nombre moyen de jours de pluie est de 55. Les pics de température mensuelle maximale sont enregistrés en mars $\left(38^{\circ} \mathrm{C}\right)$ et en novembre $\left(33{ }^{\circ} \mathrm{C}\right)$, et les pics de température mensuelle minimale surviennent aux mois de janvier $\left(17^{\circ} \mathrm{C}\right)$ et d'août $\left(15^{\circ} \mathrm{C}\right)$.

L'étude a nécessité une phase de collecte de données de base à travers une synthèse bibliographique suivie d'une enquête sur les trypanosomoses et leurs principaux vecteurs.

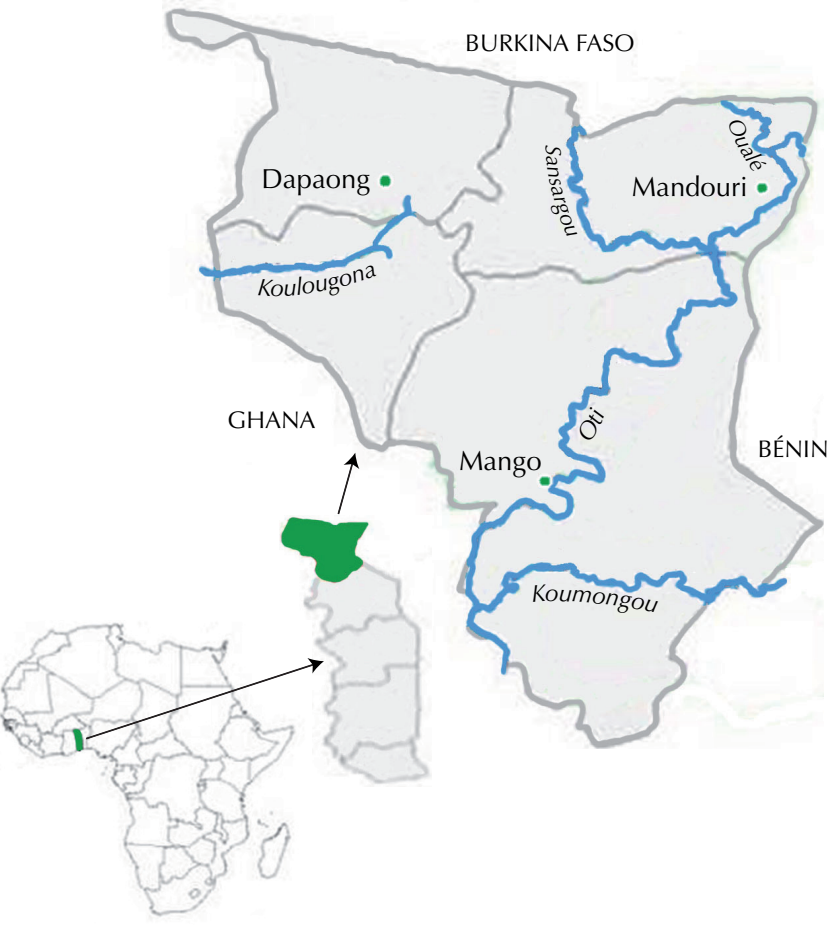

Figure 1 : zone d'étude.

\section{Synthèse bibliographique}

La documentation a comporté diverses cartes thématiques disponibles : carte de végétation, d'occupation des terres, de relief, de géologie, d'hydrographie et des voies de communication. Les couches de données alphanumériques relatives à l'agriculture, la démographie, l'élevage et les données épidémiologiques existantes ont été extraites des publications scientifiques, des rapports et mémoires. Les cartes, souvent à différentes échelles et projections, ont été numérisées puis importées à l'aide d'un système d'information géographique, Arcview GIS 3.2a. Le système de projection qui limite les déformations au niveau du Togo est la projection universal transverse mercator (UTM), fuseau 31. Le géoïde de référence est le World Geodesic System 1984 (WGS84).

\section{Enquêtes sur le vecteur et le parasite}

Deux enquêtes entomologique et protozoologique ont été menées simultanément à la fin de la saison sèche durant les mois d'avril et de mai 2006. Le fleuve Oti et ses affluents ont été prospectés avec deux types de pièges, le piège biconique standard (10) et le piège monoconique de type Vavoua (23). Dans chaque site, les pièges (biconique et monoconique posés alternativement) ont été déployés pendant 48 heures à un intervalle de 100-200 m le long de la végétation riveraine. Chaque piège a été localisé à l'aide d'un système de positionnement global (GPS). En pratique, 46, 28, 12, 6 et 3 pièges ont été posés respectivement sur le long de la rivière Oti et de ses affluents, Sansargou, Koumongou, Oualé et Koumbéloti. En plus, 30 pièges ont été déployés sur le Koulougona, un affluent de la Volta blanche. Les mouches capturées ont été dénombrées selon les espèces, le sexe et le statut nutritionnel. La densité apparente a été définie comme le nombre moyen de mouches tsé-tsé capturées par piège et par jour.

Les troupeaux échantillonnés ont été choisis au hasard parmi ceux qui ont été trouvés dans le voisinage des sites de piégeage en se basant sur une énumération obtenue des riverains. Au niveau de chaque troupeau, les bovins ont été choisis parmi les animaux présents au moment de l'échantillonnage en tenant compte de l'effectif et de la structure du troupeau. Au moins 10 p. 100 de l'effectif total a été prélevé. Les positions géographiques des troupeaux échantillonnés ont été relevées à l'aide du GPS. Les prélèvements sanguins ont été effectués à la veine jugulaire dans des tubes héparinés de $5 \mathrm{ml}$. Au total, 486 bovins ont été examinés dans 33 troupeaux, soit 79 taurins, 315 métis et 92 zébus (11). Les examens parasitologiques ont été effectués par la méthode du buffy coat (29). Le sang collecté a été soumis à une centrifugation différentielle dans les tubes capillaires. Le buffy coat a été examiné en microscopie à fond noir après son étalement entre lame et lamelle. La lecture de l'hématocrite a été effectuée sur un lecteur standard. Les différentes espèces de trypanosomes ont été identifiées par rapport à leurs mouvements. Les principaux paramètres intervenant dans le diagnostic, tels que l'âge, la race, la nature et la date du dernier traitement trypanocide et le nombre d'animaux présents, ont également été notés.

\section{Enquête sur les hôtes nourriciers sauvages et domestiques}

Cette enquête a été conduite simultanément avec les enquêtes entomologiques. Elle a consisté à évaluer, avec l'aide d'un spécialiste des eaux et forêts, l'abondance des grands mammifères sauvages et domestiques par la recherche de leurs indices de présence (crottes, empreintes, présence physique et restes de nourriture). L'état de dégradation des indices (frais, récent, vieux, très vieux) a été relevé. 


\section{RESULTATS}

\section{Aspects physiques et humains de la zone d'étude}

Lorsqu'on parle d'un état dégradé ou fragmenté, on se réfère toujours à un état original homogène. La fragmentation des habitats est un thème d'actualité sur le plan mondial, mais peu d'écrits relatifs à ce sujet, du moins en ce qui concerne la zone d'étude, sont disponibles. Néanmoins, les résultats de deux travaux, l'un sur l'état de dégradation des terres et de l'environnement au Togo (8) et l'autre sur l'inventaire des terres irrigables de la région des Savanes ont été exploités (26). En associant ces données aux critères géomorphologiques, agronomiques, humains et socioéconomiques, les auteurs ont défini trois grands ensembles homogènes (figure 2) : du nord au sud, zone I, zone II et zone III. Les caractéristiques physiques et humaines de chaque sous-zone ont été décrites dans le tableau I.

\section{Résultats entomologiques}

Deux espèces de glossines riveraines (Glossina palpalis palpalis, $G$. tachinoides) et une espèce de savane (G. morsitans submorsitans) ont été capturées. G. tachinoides a été l'unique espèce prise sur l'Oti et sur ses affluents. Aucune glossine n'a été capturée sur la rivière Koulougona. G. m. submorsitans et G. p. palpalis ont été capturées sur la rivière Koumongou au niveau de Naboulgou dans le parc national de Kéran. Les sites de capture de ces trois espèces ont été présentés dans la figure 3. Au niveau régional, la densité apparente par piège et par jour de mouches tsé-tsé a été de 0,67 \pm 0,49 (tableau II).

\section{Résultats parasitologiques}

Le diagnostic parasitologique a donné une prévalence moyenne de $3,29 \pm 1,58$ p. 100 (tableau III) de trypanosomes pathogènes dont
12 cas de Trypanosoma vivax (2,47 p. 100) et 4 cas de T. congolense (0,82 p. 100). Aucun cas d'infection mixte ou par T. brucei n'a été diagnostiqué. L'hématocrite moyen des troupeaux a été de 26,17 \pm 0,54 p. 100 pour tous les types de bovins. La moyenne la plus élevée a été de 29 p. 100 à Natchakou et la plus faible de 22 p. 100 à Mango. Le pourcentage d'individus dont l'hématocrite a été inférieur à 25 p. 100 (seuil classiquement considéré comme révélateur d'un état

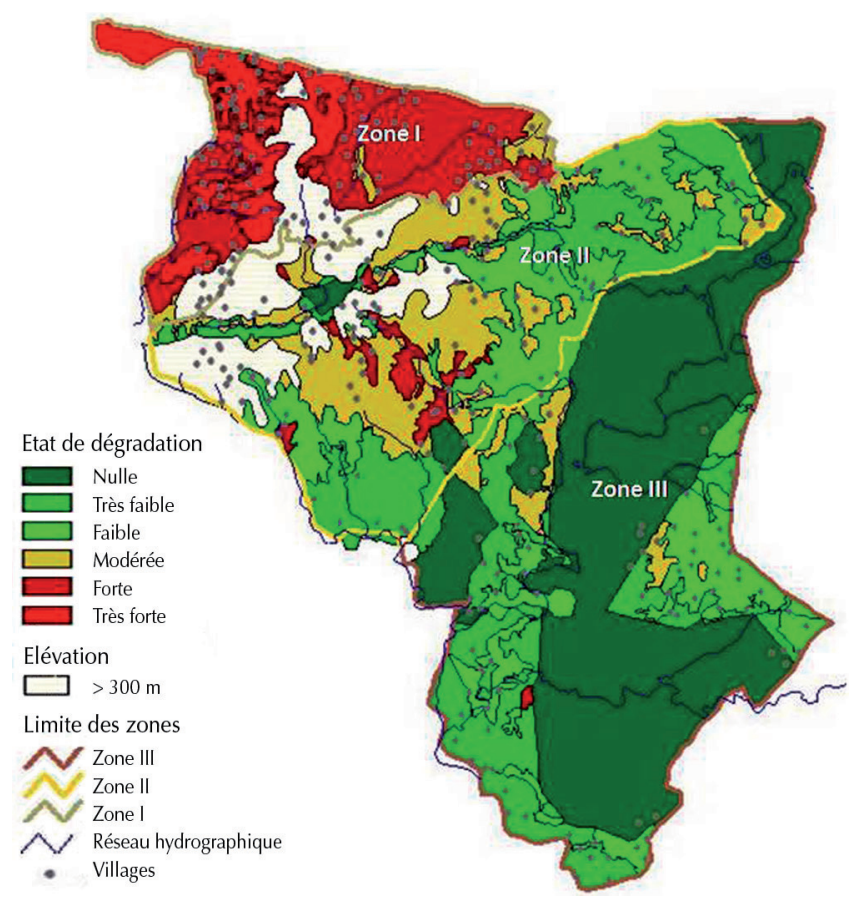

Figure 2 : ensembles homogènes du bassin.

\section{Tableau I}

Caractéristiques physiques et humaines des zones homogènes

\begin{tabular}{|c|c|c|c|}
\hline Caractéristiques & Zone I & Zone II & Zone III \\
\hline Géomorphologie & $\begin{array}{l}\text { Pénéplaine sur socle granitique } \\
\text { Sol pauvre très dégradé }\end{array}$ & $\begin{array}{c}\text { Collines gréseuses avec des falaises abruptes } \\
\text { Sol modérément dégradé }\end{array}$ & $\begin{array}{l}\text { Vaste plaine en pente douce } \\
\text { Sol marécageux et inondable }\end{array}$ \\
\hline Altitude & $200-250 \mathrm{~m}$ & $300-350 \mathrm{~m}$ & $100-200 \mathrm{~m}$ \\
\hline Pluviométrie & 900-1000 mm & $1000 \mathrm{~mm}$ & $1000-1100 \mathrm{~mm}$ \\
\hline Aires protégées & 0 & 3 & 2 \\
\hline Hydrographie & Rivières non permanentes & Rivières non permanentes & 3 rivières permanentes \\
\hline Terres utilisés (\%) & $60-80 \%$ & $10-30 \%$ & $<10 \%$ \\
\hline Densité humaine & $>200$ hab. $/ \mathrm{km}^{2}$ & 25-100 hab. $/ \mathrm{km}^{2}$ & 10-20 hab. / km² \\
\hline Densité bétail & $>25$ têtes / km² & 10-25 têtes / km² & 5-10 têtes / km² \\
\hline Mouvement migratoire & re Vers les zones II et III & Vers la zone III & Zone d'accueil \\
\hline Transhumance & Petite transhumance & Petite transhumance & Zone d'accueil \\
\hline Mode d'élevage & $\begin{array}{c}\text { Traditionnel } \\
\text { à but non commercial } \\
\text { Race taurine très représentée }\end{array}$ & $\begin{array}{c}\text { Traditionnel } \\
\text { à but commercial } \\
\text { Race taurine très représentée }\end{array}$ & $\begin{array}{l}\text { Traditionnel amélioré } \\
\text { à but commercial } \\
\text { Un fort taux de métissage } \\
\text { avec les zébus }\end{array}$ \\
\hline Traction animale & Très utilisée & Moyennement utilisée & Faiblement utilisée \\
\hline Principales cultures & $\begin{array}{l}\text { Culture vivrière } \\
\text { (céréale, légumineuse) } \\
\text { Culture de rente (coton) }\end{array}$ & Culture de rente (coton) & $\begin{array}{l}\text { Culture vivrière (céréale, } \\
\text { légumineuse, tubercule) } \\
\text { Culture de rente (coton, pastèque *) }\end{array}$ \\
\hline
\end{tabular}

* Culture récemment introduite par les immigrants Mossé 


\section{Tableau II}

Densité de glossines capturées par site

\begin{tabular}{|c|c|c|c|c|c|c|c|c|c|c|c|c|c|c|c|c|c|c|c|c|c|}
\hline \multirow[t]{3}{*}{ Site de piégeage } & \multirow{3}{*}{$\begin{array}{l}\text { Nb. de } \\
\text { pièges }\end{array}$} & \multirow{3}{*}{$\begin{array}{l}\text { Nb. de } \\
\text { jours }\end{array}$} & \multicolumn{6}{|c|}{ Glossina tachinoides } & \multicolumn{6}{|c|}{ Glossina p. palpalis } & \multicolumn{6}{|c|}{ G. m. submorsitans } & \multirow[t]{3}{*}{ DAP } \\
\hline & & & \multirow{2}{*}{\multicolumn{3}{|c|}{$\frac{M}{\text { T NT TM }}$}} & \multicolumn{3}{|c|}{$\mathbf{F}$} & \multicolumn{3}{|c|}{ M } & \multicolumn{3}{|c|}{$F$} & \multicolumn{3}{|c|}{$M$} & \multicolumn{3}{|c|}{$F$} & \\
\hline & & & & & & & NT & TF & $\mathbf{T}$ & NT & TM & $\mathbf{T}$ & NT & TF & $\mathbf{T}$ & NT & $\mathrm{TM}$ & $\mathbf{T}$ & NT & TF & \\
\hline $\begin{array}{l}\text { Tambango } \\
\text { (Fosse aux lions) }\end{array}$ & 10 & 2 & 0 & 0 & 0 & 0 & 0 & 0 & 0 & 0 & 0 & 0 & 0 & 0 & 0 & 0 & 0 & 0 & 0 & 0 & 0,00 \\
\hline Nano - Moane & 10 & 2 & 0 & 0 & 0 & 0 & 0 & 0 & 0 & 0 & 0 & 0 & 0 & 0 & 0 & 0 & 0 & 0 & 0 & 0 & 0,00 \\
\hline Tampialime & 10 & 2 & 0 & 0 & 0 & 0 & 0 & 0 & 0 & 0 & 0 & 0 & 0 & 0 & 0 & 0 & 0 & 0 & 0 & 0 & 0,00 \\
\hline Pogno & 2 & 2 & 0 & 0 & & 0 & 0 & 0 & 0 & 0 & 0 & 0 & 0 & & 0 & 0 & 0 & 0 & 0 & 0 & 0,00 \\
\hline Borgou - Bombenga & 20 & 2 & 0 & 0 & 0 & 0 & 0 & 0 & 0 & 0 & 0 & 0 & 0 & 0 & 0 & 0 & 0 & 0 & 0 & 0 & 0,00 \\
\hline Panseri - Bouldjoaré & 12 & 2 & 12 & 6 & 18 & 14 & 16 & 30 & 0 & 0 & 0 & 0 & 0 & 0 & 0 & 0 & 0 & 0 & 0 & 0 & 2,00 \\
\hline Taro & 12 & 2 & 4 & 19 & 23 & 6 & 12 & 18 & 0 & 0 & 0 & 0 & 0 & 0 & 0 & 0 & 0 & 0 & 0 & 0 & 1,71 \\
\hline Donga - Mandouri & 8 & 2 & 2 & 7 & 9 & 0 & 3 & 3 & 0 & 0 & 0 & 0 & 0 & 0 & 0 & 0 & 0 & 0 & 0 & 0 & 0,78 \\
\hline Nagbati & 6 & 2 & 0 & 1 & 1 & 0 & 1 & 1 & 0 & 0 & 0 & 0 & 0 & 0 & 0 & 0 & 0 & 0 & 0 & 0 & 0,17 \\
\hline D. Graviwou & 14 & 2 & 1 & 1 & 2 & 0 & 3 & 3 & 0 & 0 & 0 & 0 & 0 & 0 & 0 & 0 & 0 & 0 & 0 & 0 & 0,18 \\
\hline Mango & 8 & 2 & 0 & 0 & 0 & 0 & 0 & 0 & 0 & 0 & 0 & 0 & 0 & 0 & 0 & 0 & 0 & 0 & 0 & 0 & 0,00 \\
\hline Gnagbandi & 4 & 2 & 0 & 2 & 2 & 0 & 0 & 0 & 0 & 0 & 0 & 0 & 0 & 0 & 0 & 0 & 0 & 0 & 0 & 0 & 0,25 \\
\hline Naboulgou & 12 & 2 & 26 & 17 & 43 & 0 & 2 & 2 & 0 & 0 & 0 & 1 & 0 & 1 & 0 & 1 & 1 & 3 & 12 & 15 & 2,58 \\
\hline Moyenne régionale & & & & & & & & & & & & & & & & & & & & & 0,67 \\
\hline Ecart-type & & & & & & & & & & & & & & & & & & & & & \\
\hline $\begin{array}{l}\text { Intervalle } \\
\text { de confiance }\end{array}$ & & & & & & & & & & & & & & & & & & & & & \\
\hline
\end{tabular}

M : mâle ; F : femelle ; T : ténéral ; NT : non ténéral ; TM : total mâles ; TF : total femelles ; DAP : densité apparente/piège/jour $* \alpha=0,05$

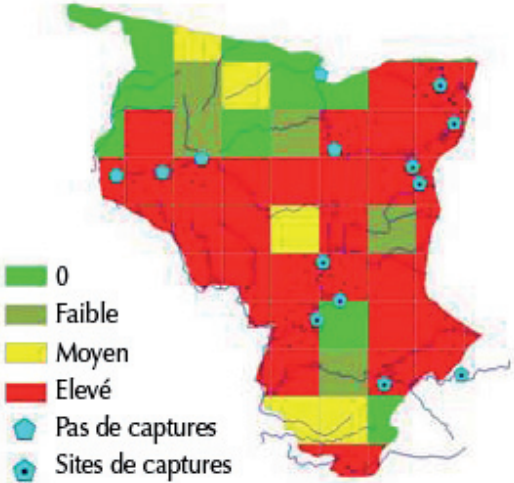

a) Glossina tachinoides

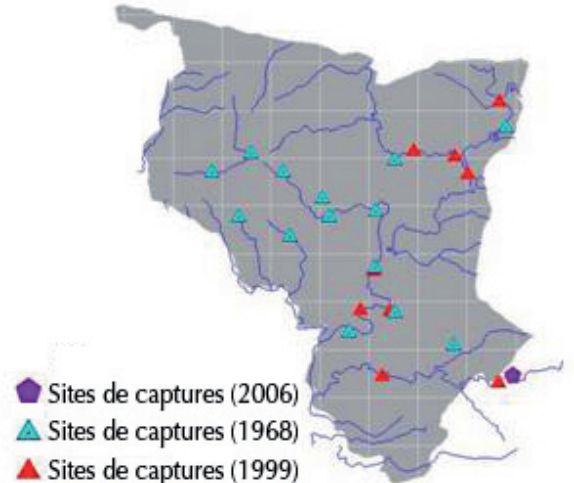

b) G. palpalis palpalis

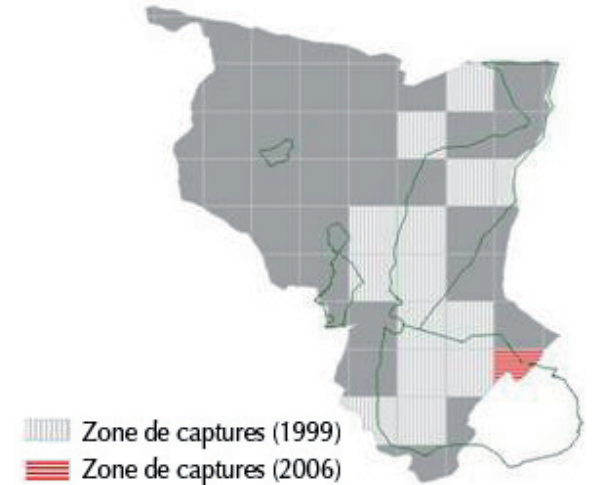

c) G. morsitans submorsitans

Figure 3 : carte de répartition des glossines. Source : Hendrickx et Napala, 1999.

pathologique) a été de 28 p. 100, soit 136/486. Ce pourcentage a été de 29, 30 et 22 p. 100 respectivement pour les taurins (23/79), les métis $(93 / 315)$ et les zébus (22/92). Les rapports entre les prévalences et les hématocrites obtenus dans la présente étude et ceux provenant de résultats antérieurs sont présentés dans la figure 4.

\section{DISCUSSION}

Les résultats de cette étude ont montré que $G$. tachinoides a été la seule espèce de glossine capturée sur l'Oti et ses affluents au nord de $10^{\circ}$ de latitude nord. Quant aux deux autres espèces,
G. m. submorsitans et G. p. palpalis, elles n'ont été prises que sur la rivière Koumongou. En revanche, sur la rivière Koulougona traversant la réserve de faune de la Fosse aux lions, aucune glossine n'a été capturée. La carte de distribution des glossines (figure 3) a montré un important changement de la distribution spatiale (limite Nord) des espèces capturées par rapport aux résultats précédents $(11,17,18,19,21,24,25)$. Jusqu'en 1999, G. p. palpalis (figure 3b) a été souvent capturée au niveau des îlots de végétation riveraine dense associés à une eau stagnante au nord de $10^{\circ}$ de latitude nord. Quant à G. m. submorsitans (figure 3c) souvent associée à la faune sauvage, elle a été capturée tout le long de l'Oti, de l'embouchure de Koumongou 


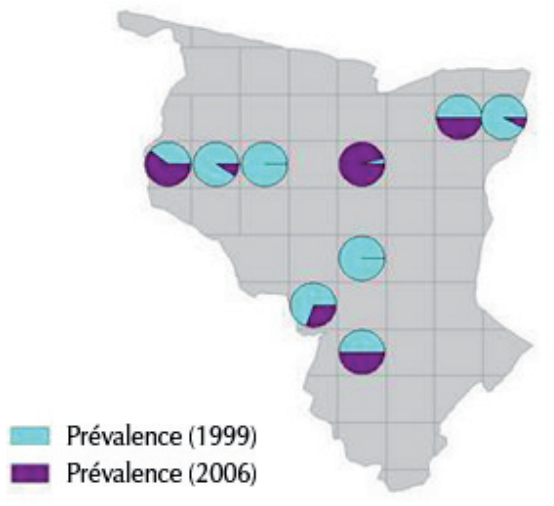

a) Prévalence

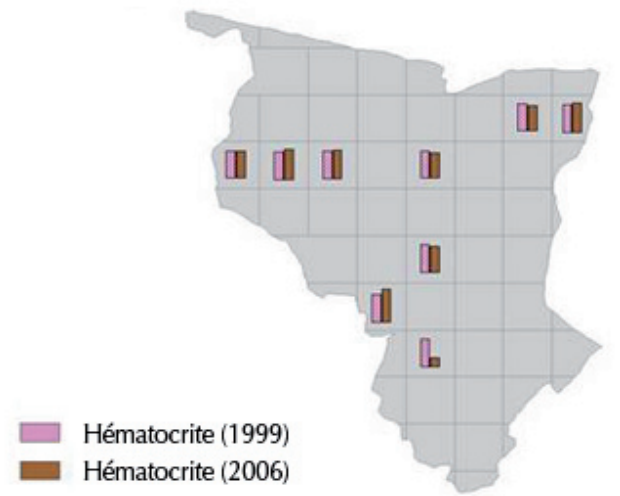

b) Hématocrite
Figure 4 : rapport entre les prévalences et les hématocrites obtenus en 1999 et 2006.

\section{Tableau III}

Prévalences trypanosomiennes et hématocrite des bovins par localité

\begin{tabular}{|c|c|c|c|c|c|c|c|c|c|c|c|}
\hline Localité & $\begin{array}{c}\text { Taille } \\
\text { échantillon }\end{array}$ & Hct & SD & IC & TC & TV & TB & $\begin{array}{c}\text { Prévalence } \\
(\%)\end{array}$ & SD & IC & $\begin{array}{c}\text { Nb. bovins } \\
\text { Hct }<25\end{array}$ \\
\hline Tambango & 24 & 28 & & & 0 & 0 & 0 & 0 & 3 & & 3 \\
\hline Moane & 35 & 26 & & & 2 & 0 & 0 & 5,71 & 17 & & 17 \\
\hline Nano & 50 & 27 & & & 0 & 0 & 0 & 0 & 10 & & 10 \\
\hline Tampialime & 35 & 27 & & & 2 & 2 & 0 & 11,43 & 7 & & 7 \\
\hline Borgou & 17 & 27 & & & 0 & 0 & 0 & 0 & 4 & & 4 \\
\hline Bombenga & 33 & 27 & & & 0 & 0 & 0 & 0 & 9 & & 9 \\
\hline Bouldjoaré & 34 & 25 & & & 0 & 0 & 0 & 0 & 13 & & 13 \\
\hline Mandouri & 50 & 27 & & & 0 & 2 & 0 & 4 & 15 & & 15 \\
\hline Donga & 63 & 26 & & & 0 & 2 & 0 & 3,17 & 32 & & 32 \\
\hline Mango & 64 & 22 & & & 0 & 3 & 0 & 4,69 & 17 & & 17 \\
\hline Natchakou & 18 & 29 & & & 0 & 0 & 0 & 0 & 1 & & 1 \\
\hline N'gongopri & 15 & 27 & & & 0 & 0 & 0 & 0 & 3 & & 3 \\
\hline Massangou & 17 & 27 & & & 0 & 0 & 0 & 0 & 4 & & 4 \\
\hline Akpossou & 31 & 28 & & & & & & 9,68 & 1 & & 1 \\
\hline Moy. rég. & & 26,17 & 6,18 & 0,55 & & & & 3,29 & 17,86 & 1,59 & \\
\hline Taurin & 79 & 27 & & & 1 & 2 & 0 & 3,8 & & & 23 \\
\hline Zébu $x$ taurin & 315 & 26 & & & 3 & 6 & 0 & 2,86 & & & 93 \\
\hline Zébu & 92 & 27 & & & 0 & 4 & 0 & 4,35 & & & 20 \\
\hline
\end{tabular}

Hct : hématocrite ; SD : écart-type ; IC : intervalle de confiance ; TC : Trypanosoma congolense : TV : Trypanosoma vivax : TB : Trypanosoma brucei ; Moy. rég. : moyenne régionale

au sud de $10^{\circ}$, jusqu'au-delà de $11^{\circ}$ de latitude nord, au nordest du bassin versant. En ce qui concerne G. tachinoides, les résultats des travaux antérieurs ont montré son omniprésence sur tout le réseau hydrographique du bassin versant de l'Oti (figure 3a). Les résultats ont montré que, bien que prédominante (90 p. 100 des captures soit 155/172), G. tachinoides n'a pas été capturée sur la rivière Koulougona. Sur la rivière Sansargou, elle n'a été capturée qu'au niveau de la confluence avec l'Oti. Quant à G. p. palpalis et G. m. submorsitans, elles ont été sous le seuil mesurable dans le parc national Oti-Mandouri.

Bien que moins efficaces pour les glossines de savanes, les pièges biconiques Challier - Laveissière et les pièges monoconiques de type Vavoua, utilisés dans cette étude, sont des pièges standard, très reconnus pour leur efficacité à capturer des glossines riveraines. Ainsi, la méthode utilisée ne pouvait pas être incriminée pour justi- fier la disparition de G. p. palpalis. Néanmoins, la durée de capture des glossines $(48 \mathrm{~h})$ était probablement trop courte pour juger définitivement de l'absence de glossines dans une zone donnée. Une durée de 72 à 94 h répétée dans différents gîtes et à différentes saisons serait souhaitable.

Les actions anthropiques locales et le climat ont été les facteurs déterminants de la réduction de la répartition spatiale et de l'abondance des glossines. En effet, depuis 1990, les aires protégées ont été totalement ou partiellement envahies par les agriculteurs et éleveurs riverains auparavant chassés de force. Ces hommes, à la recherche de terres arables, de bois de chauffe et de charbon de bois, ont défriché de grandes surfaces et détruit des arbres. Ils ont aussi abattu la faune. L'extermination drastique de la faune dans la région des Savanes est rapportée par Schneider (32) qui écrit : «qu'après que les premiers animaux sauvages aient été tués sans 
que les auteurs ne soient punis, un vrai massacre de la faune s'était enclenché ». Privées ainsi de leurs habitats et de leurs sources nourricières, les glossines ont régressé ou disparu. Comme d'autres auteurs l'ont déjà souligné $(13,19,20,30,31)$, la disparition ou la régression des mouches de savanes a ainsi accompagné la flambée du braconnage et la réinstallation des agriculteurs dans les zones protégées. Le retour des agriculteurs et le braconnage ont provoqué une baisse du nombre des animaux sauvages, une augmentation des surfaces cultivées et une dégradation des habitats naturels des glossines. Les espèces de glossines du groupe morsitans ont été les premières victimes et les plus vulnérables à l'accroissement de la population humaine et des surfaces agricoles. Cependant, Hendrickx et coll. $(17,19,20)$ ont trouvé de faibles densités de $G$. tachinoides dans les zones où l'intensité de l'agriculture était élevée. Les auteurs de la présente étude ont fait le même constat dans la réserve de la Fosse aux lions où le massacre des animaux sauvages a atteint son paroxysme. Aucune G. tachinoides n'a été capturée.

Le climat est un des facteurs de la régulation des populations de glossines, ces dernières ayant des exigences bien définies, même dans leurs propres zones de distribution (22). Les climats chauds et excessivement secs limitent leur expansion. Les enquêtes ont eu lieu en saison sèche chaude, période où les mouches tsé-tsé se concentrent dans leurs gîtes favorables. Des spécialistes ont montré que, depuis 1961, le climat de la région des Savanes du Togo tend à une aridification. Ils ont noté une augmentation de la température moyenne annuelle de $0,8{ }^{\circ} \mathrm{C}$ et une diminution de la pluviosité de $2,22 \mathrm{~mm} / \mathrm{an}$ et de 10,6 jours de pluie (27). D'octobre 2005 à mai 2006, la région a connu huit mois secs (pluviométrie $<30 \mathrm{~mm} / \mathrm{mois}$ ). Ce déficit pluviométrique associé aux températures déjà élevées a favorisé le réchauffement, le tarissement des cours d'eaux et des sols provoquant certainement une grande mortalité des pupes enfouies. Cette situation a pu mener à des densités de $G$. p. palpalis en dessous du seuil mesurable au niveau de la rivière Oti et a concerné toutes les espèces dans la réserve de la Fosse aux lions.

Un troisième facteur non moins important qui a pu contribuer à la régression des glossines dans le BVO a été la campagne anti-tsé-tsé menée par le Projet national de lutte contre les trypanosomoses et leurs vecteurs durant sa phase d'exécution entre 1990 et 1999. Au cours de celle-ci, des essais pilotes d'application d'insecticide sur bétail (imprégnation topique et pulvérisation) à grande échelle ont été réalisés. En outre, entre les années 1997 et 1999, douze communautés rurales des zones classées prioritaires ont été organisées afin de mener de manière autonome des applications collectives, synchrones et séquentielles d'insecticide sur du bétail. Cette campagne a réduit les glossines en dessous du seuil mesurable (5). Tous ces facteurs ont contribué à la chute de la densité des glossines réduisant ainsi le contact entre ces dernières et les bovins.

De faibles prévalences parasitologiques de trypanosomoses (0-5 p. 100) ont été observées dans les sites enquêtés à l'exception de deux sites, Akpossou (9 p. 100) et Tampialime (11 p. 100). Contrairement aux travaux antérieurs réalisés par Dao (11), et Hendrickx et coll. $(17,19,20)$, les présents résultats ont révélé l'ascendance de T. vivax sur T. congolense. Ce changement a été certainement dû à la raréfaction de G. m. submorsitans qui est un vecteur de T. congolense (Djiteye, 2008, commun. pers.). Les vecteurs mécaniques permettent un relais de la transmission de $T$. vivax. Il serait judicieux qu'une étude sur ces vecteurs mécaniques soit menée afin de vérifier cette hypothèse. A l'exception du site de Tampialiame à l'extrême ouest de la zone d'étude où la prévalence a été plus élevée que celle des travaux antérieurs $(11,19)$ dans la plupart des cas, le rapport entre les deux prévalences a été en faveur de celle obtenue antérieurement (figure 4a). La faible prévalence obtenue pouvait aussi s'expliquer par la sensibilité de la méthode utilisée. En effet, la méthode du buffy coat n'est pas très sensible pour mesurer la prévalence des infections dans les situations d'enzootie (14). De plus la sélection extemporanée des animaux exposait au risque d'utiliser des animaux traités avec des chimiopréventifs, réduisant les prévalences observées. Néanmoins, associée à la valeur de l'hématocrite, elle a permis d'estimer l'importance des cas de trypanosomose à environ 28 p. 100 (tableau III). Comme toutes les autres techniques parasitologiques, elle est mieux indiquée en zone d'épizootie. Une analyse sérologique s'avère indispensable afin de compléter et d'améliorer ces résultats.

Les hématocrites moyens calculés ont été supérieurs ou égaux à 25 p. 100 dans tous les sites à l'exception de Mango, mais ils ont été concentrés autour de 27 p. 100. Ces hématocrites n'ont pas varié comparés à ceux obtenus antérieurement (figure 4b), ce qui est étonnant vue la réduction observée des prévalences parasitaires et des densités apparentes/piège/jour. D'autres facteurs anémiant tels que les helminthes, les hémoparasites autres que les trypanosomes et le déficit nutritionnel ont pu contribuer au maintien de ces hématocrites.

\section{CONCLUSION}

Cette étude a montré qu'il y a eu une modification très sensible dans la répartition géographique des glossines dans le Nord Togo. Ceci est observé surtout pour G. m. submorsitans et G. p. palpalis. La limite nord de ces glossines se localise maintenant nettement plus au sud où subsistent encore leurs habitats naturels, leurs hôtes et l'eau sur une grande partie de l'année. Cette modification de la répartition géographique des glossines a été provoquée indirectement par l'homme, par son action sur la nature (braconnage et déforestation) et aussi par la sécheresse que connaît la région ces derniers temps. Le recul et la baisse de la densité des glossines ont réduit le contact entre ces dernières et les hôtes domestiques, en particulier les bovins, ce qui s'est traduit par les faibles prévalences obtenues. La non ré-invasion de la partie nord du bassin de l'Oti à partir du sud pourrait être due au changement climatique. En effet, ces résultats renforcent l'hypothèse que, dans certains cas, l'effet combiné d'un climat en général plus aride et d'une pression importante de l'agriculture sur le biotope naturel des glossines contribue à empêcher la réinstallation de ces populations après des opérations de contrôle $(16,17)$ au niveau de leur limite nord en Afrique de l'Ouest. Dans d'autres cas cependant, les zones libérées ont été envahies à nouveau (12). Il serait souhaitable d'étudier ce phénomène avant la lutte, notamment en utilisant les outils génétiques qui permettent de mesurer les probabilités de ré-invasion (7) avec des conséquences importantes sur la méthode générale à adopter pour éradiquer progressivement ce fléau de la zone en partant de la limite nord et avec des risques de ré-invasion minimaux.

\section{Remerciements}

Ce travail a été réalisé dans le cadre du projet FSP, financé par le ministère des Affaires étrangères de la France. Les auteurs remercient Pr A. Gouro, directeur général du Cirdes, pour avoir créé de bonnes conditions de travail, et Dr Z. Bengaly, chef de l'Urbio. Ils remercient les responsables de l'Institut togolais de recherche agronomique (Itra), en particulier Dr A. Agbobli, directeur général, Dr K. Adomefa, directeur scientifique, et M. B. Bonfoh, directeur du CRA-SH. Ils adressent aussi leurs remerciements à D. Amédji, S. Yacnambé, K. Tsini, D. Tchassanti, I. Soulé, K. Awator et K.J. Sanou. 


\section{BIBLIOGRAPHIE}

1. ADDRA T.C., FAHEM A.K., DE JONG T., MANK T., 1984. Atlas du développement régional du Togo. Lomé, Togo, ministère du Plan et de I'Industrie, direction générale du Plan et du Développement, 207 p.

2. ASSI H., 2004. Définition d'une stratégie de revalorisation d'un écosystème fragile : cas de la forêt classée de Sadji (Togo). Mémoire Etudes spécialisées en gestion des ressources animales et végétales en milieux tropicaux, Université de Liège, Belgique, $77 \mathrm{p}$.

3. AUBREVILLE A., 1937. Les forêts du Dahomey et du Togo. Bull. Com. Etud. hist. sci. AOF, 20.

4. BANKATI W., 1996. Etude des systèmes d'élevage du bétail dans les zones à glossines récemment occupées par les paysans : cas de la vallée de l'Oti (région des Savanes). Mémoire Ingénieur Agronome, Ecole supérieure d'Agronomie, Université du Bénin, Lomé, Togo, 131 p.

5. BASTIAENSEN P., KOUAGOU N.T., GNOFAM M., BATAWUI K., NAPALA A., HENDRICKX G., 2004. Adoption d'une nouvelle technique de contrôle de la mouche tsétsé par des éleveurs du nord du Togo : considération socioéconomiques. Bull. Anim. Health Prod. Afr., 52 : 142-158.

6. BOUYER J., 2006. Ecologie des glossines du Mouhoun au Burkina Faso : intérêt pour l'épidémiologie et le contrôle des trypanosomoses africaines. Thèse Doct. Sci. chim. biol. Santé, Université de Montpellier II, France, $204 \mathrm{p}$.

7. BOUYER J., RAVEL S., DUJARDIN J.P., DE MEES T., VIAL L., THEVENON S., GUERRINI L., SIDIBE I., SOLANO P., 2007. Population structuring of Glossina palpalis gambiensis (Diptera: Glossinidae) according to landscape fragmentation in the Mouhoun River, Burkina Faso. J. med. entomol., 44: 788-795.

8. BRABANT P., DARRACQ S., EGUE K., SIMONNEAUX V., 1996. Etat de dégradation des terres résultant des activités humaines. Paris, France, Orstom, $47 \mathrm{p}$.

9. CHALLIER A., 1973. Ecologie de Glossina palpalis gambiensis Vanderplank, 1949 (Diptera, Muscidae) en savane d'Afrique Occidentale. Paris, France, Orstom, $274 \mathrm{p}$.

10. CHALLIER A., LAVEISSIERE C., 1973. Un nouveau piège pour la capture des glossines (Glossina : Diptera, Muscidae) : description et essai sur le terrain. Cah. Orstom. Sér. Ent. méd. parasitol., 11 : 252-262.

11. DAO B.B., 1998. Trypanosomose et trypanotolérance au Togo : contribution du système d'information géographique (SIG) à l'étude de I'introgression zébu. Mémoire DEA, faculté des Sciences et Techniques, Université de Dakar, Sénégal, 56 p.

12. DE LA ROCQUE S., MICHEL J.F., BOUYER J., DE WISPELAERE G., CUISANCE D., 2005. Geographical information systems in parasitology: a review of potential applications using the example of animal trypanosomosis in West Africa. Parasitologia, 47: 97-104.

13. DE LA ROCQUE S., MICHEL J.F., CUISANCE D., DE WISPELAERE G., SOLANO P., AUGUSSEAU X., ARNAUD M., GUILLOBEZ S., 2001. Du satellite au microsatellite, le risque trypanosomien : une approche globale pour une décision locale. Montpellier, France, Cirad, 151 p.

14. DESOUESNES M., MICHEL J.-F., DE LA ROCQUE S., SOLANO P., MILLOGO L., BENGALY Z., SIDIBE I., 1999. Enquête parasitologique et sérologique (Elisa-indirect) sur les trypanosomoses des bovins dans la zone de Sidéradougou, Burkina Faso. Revue Elev. Méd. vét. Pays trop., $52: 223-232$.

15. FOURY P., 1948. Principes des sylvicultures tropicales. Bois Forêts Trop., $2: 7 \mathrm{p}$.

16. HENDRICKX G., DE LA ROCQUE S., MATTIOLI R., 2004. Towards long-term tsetse and trypanosomosis management options in West Africa. Rome, Italy, FAO, 57 p. (PAAT tech. sci. Ser. 6)
17. HENDRICKX G., DE LA ROCQUE S., REID R., WINT W., 2001. Spatial trypanosomosis management. From data-layers to decision making. Trends Parasitol., 17: 35-42.

18. HENDRICKX G., NAPALA A., 1999. Le contrôle de la trypanosomose " à la carte »: une approche intégrée basée sur un système d'information géographique. Acad. R. Sci. Outre-Mer, $90 \mathrm{p}$.

19. HENDRICKX G., NAPALA A., DAO B., BATAWUI D., DE DEKEN R., VERMEILEN A., SLINGENBERGH J.H.W., 1999. A systematic approach to area-wide tsetse distribution and abundance maps. Bull. Entomol. Res., 89: 231-244.

20. HENDRICKX G., ROGERS D.J., NAPALA A., SLINGENBERGH J.H.W., 1995. Predicting the distribution of riverine tsetse and the prevalence of bovine trypanosomosis in Togo using ground-based and satellite data. In: ISCTRC, Kampala, Uganda, 1993. Nairobi, Kenya, OUA-STRC.

21. ITARD J., 1968. Enquête entomologique dans la région des Savanes (République du Togo). Revue Elev. Méd. vét. Pays trop., 21 : 375-385.

22. LAVEISSIERE C., 1976. Répartition des glossines en Haute-Volta : effet de la grande sécheresse de 1972-1973. Cah. Orstom. Sér. Ent. méd. parasitol., $14:$ 293-299.

23. LAVEISSIERE C., GREBAUT P., 1990. The trapping of tsetse flies (Diptera: Glossinidae) Improvement of a model: the Vavoua trap. Trop. Med. Parasitol., 41: 185-192.

24. MAWUENA K., 1988. Données historiques et bibliographiques sur les glossines et les trypanosomiases animales au Togo (1894-1972). Trypanotol. Prod. Anim., 5 : 101-105.

25. MAWUENA K., 1988. Les glossines au Togo de 1972 à 1987 : revue de la situation et synthèse. Trypanotol. Prod. Anim., 5 : 131-135.

26. MDRHV, 1996. Inventaire de terres irrigables de la région des Savanes. Lomé, Togo, Mdrhv, 35 p.

27. MERF, 2001. Plan national d'action pour l'environnement. Lomé, Togo, Merf, $173 \mathrm{p}$.

28. MERF, 2001. Programme d'action national de lutte contre la désertification. Lomé, Togo, Merf, 161 p.

29. MURRAY M., MURRAY P.K., MCINNTYE W.I.M., 1974. An improved parasitological technique for diagnosis of African trypanosomiasis. Trans. R. Soc. trop. Med. Hyg., 71: 325-326.

30. RAWLINGS P., CEESAY M.L., WACHER T.J., SNOW W.F., 1993. The distribution of the tsetse flies Glossina morsitans submorsitans and G. palpalis gambiensis (Diptera: Glossinidae) in the Gambia and the application of survey results to tsetse and trypanosomiasis control. Bull Ent. Res., 83: 625-632.

31. REID R.S., KRUSKA R.L., DEICHMANN U., THORNTON P.K., LEAK S.G.A., 2000. Human population growth and the extinction of the tsetse fly. Agric. Ecosys. Environ., 77: 227-236.

32. SCHNEIDER K., 2001. La démocratie a fait disparaître les animaux sauvages dans le pays Moba au Togo. In : Atelier régional «Faune sauvage et bétail : complémentarité, coexistence ou compétition ? », Niamey, Niger, 16-19 janv. 2001. http://www.virtualcentre.org/fr/res/int/ atelier_niamey/default.htm\#contrib

33. TCHAMIE T.T.K., 1994. Enseignements à tirer de I'hostilité des populations locales à l'égard des aires protégées au Togo. Revue int. Forêts Ind. for., 45 : 1-8.

Reçu le 14.05.2008, accepté le 06.07.2009 


\section{Summary}

Dao B., Hendrickx G., Sidibé I., Belem A.M.G., De La Rocque S. Impact of Drought and Degradation of Protected Areas on the Distribution of Bovine Trypanosomoses and their Vectors in the Oti Catchment Basin of Northern Togo

Located at the transition of the semiarid area in the North and the humid area in the South, the Oti catchment basin (OCB) has been subjected to high anthropic and climatic pressures from the 1970s to the 1990s. Drought, advancing desertification and economic activities, which rely mainly on crops and animal husbandry, contributed to the deterioration of the basin. In addition, because of its location, OCB is visited by transhumant cattle from Burkina Faso and Niger every year during the dry season. This seasonal movement of transhumant cattle engenders transmission and upholding of transboundary animal diseases, in particular bovine trypanosomoses. Bibliographical and field data, collected at the end of the dry season in April and May 2006, and processed by a GIS, allowed to assess the impact of anthropic and climatic factors on the epidemiology of animal trypanosomoses in OCB. Results of the survey showed that OCB was badly degraded; large wild mammals and Glossina species have become almost extinct in the wildlife reserve of the Lion's Den. Glossina tachinoides was the only species captured north of $10^{\circ}$ latitude $\mathrm{N}$. Trypanosoma vivax was listed as the main parasite responsible for bovine trypanosomosis. It was concluded that land encroachment by man and climate change contributed to the southward retreat of the distribution area of Glossina species.

Keywords: Cattle - Glossina - Trypanosomosis - Drought Deforestation - Geographical information system - Togo.

\section{Resumen}

Dao B., Hendrickx G., Sidibé I., Belem A.M.G., De La Rocque S. Impacto de la sequía y de la degradación de las áreas protegidas sobre la repartición de los tripanosomas bovinos y de sus vectores en la meseta del Oti, al norte de Togo

La meseta de Oti (BVO), situada en el área de transición entre las zonas semiárida al norte y húmeda al sur, ha sufrido durante las décadas de 1970-1990 fuertes presiones antrópicas y climáticas. La sequía, el avance del desierto y las actividades económicas, que reposan esencialmente sobre la agricultura y la cría han contribuido a la degradación de esta meseta. Por otro lado, debido a su localización, la BVO recibe cada año durante la estación seca, ganado trashumante proveniente de Burkina Faso y de Níger. Este movimiento estacional de los bovinos trashumantes ha sido fuente de mantenimiento y de transmisión de varias enfermedades transfronterizas y de las tripanosomosis animales. Mediante datos de síntesis bibliográfica y encuestas de campo efectuadas al final de la estación seca, de abril a mayo 2006, tratadas con un sistema de información geográfica (SIG), se logró la evaluación del impacto de los factores antrópicos y climáticos sobre la epidemiología de las tripanosomosis animales en la BVO. Los resultados del estudio muestran que la BVO se encuentra fuertemente degradada; los grandes mamíferos salvajes y las glosinas han casi desaparecido de la reserva de fauna de la fosa de los leones. Glossina tachinoides fue la única especie capturada al norte del paralelo 10 de latitud norte. Trypanosoma vivax se clasificó como el principal parásito responsable de las tripanosomosis bovinas. Se concluyó que el hombre, mediante su efecto sobre el medio ambiente y los cambios climáticos, ha provocado una disminución hacia el sur del área de distribución de las glosinas.

Palabras clave: Ganado bovino - Glossina - Tripanosomosis Sequía - Deforestación - Sistema de información geográfica Togo. 